\title{
A numerical method for the fractional Fitzhugh-Nagumo monodomain model
}

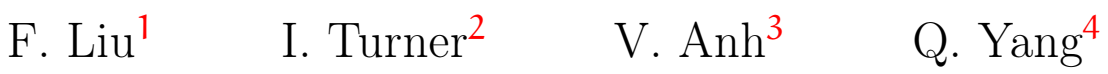 \\ K. Burrage ${ }^{5}$
}

(Received 8 November 2012; revised 9 September 2013)

\begin{abstract}
A fractional FitzHugh-Nagumo monodomain model with zero Dirichlet boundary conditions is presented, generalising the standard monodomain model that describes the propagation of the electrical potential in heterogeneous cardiac tissue. The model consists of a coupled fractional Riesz space nonlinear reaction-diffusion model and a system of ordinary differential equations, describing the ionic fluxes as a function of the membrane potential. We solve this model by decoupling the space-fractional partial differential equation and the system of ordinary differential equations at each time step. Thus, this means treating the fractional Riesz space nonlinear reaction-diffusion model as if the nonlinear source term is only locally Lipschitz. The fractional Riesz
\end{abstract}

http://journal.austms.org.au/ojs/index.php/ANZIAMJ/article/view/6372 gives this article, (c) Austral. Mathematical Soc. 2013. Published September 30, 2013, as part of the Proceedings of the 16th Biennial Computational Techniques and Applications Conference. ISSN 1446-8735. (Print two pages per sheet of paper.) Copies of this article must not be made otherwise available on the internet; instead link directly to this URL for this article. 
space nonlinear reaction-diffusion model is solved using an implicit numerical method with the shifted Grünwald-Letnikov approximation, and the stability and convergence are discussed in detail in the context of the local Lipschitz property. Some numerical examples are given to show the consistency of our computational approach.

Subject class: 26A33; 35R11; 65M12

Keywords: fractional FitzHugh-Nagumo monodomain

\section{Contents}

1 Introduction

C609

2 An implicit numerical method

C613

3 Stability and convergence

C616

4 Numerical results

C619

5 Discussion and Conclusions

C624

References

C625

\section{Introduction}

The computation of electrical wave propagation in the heart is one of the most important recent applications of mathematical modelling in physiology. Electrophysiological models of the heart describe how electrical currents flow through the heart, controlling its contractions, and are used to ascertain the effects of certain drugs designed to treat, for example, arrhythmia.

Models of the electrophysiology of a single cell are typically systems of ordinary differential equations (ODEs), while models of the propagation of 
the action potential in tissue are typically one or more partial differential equations (PDEs) - the so-called bidomain model. Numerically, the bidomain equations are challenging and it is therefore common to seek simplifications, to a so-called monodomain model. In our monodomain model, the propagation of the transmembrane potential $V_{m}(1)$, is coupled to the system of ordinary differential equations (2), which describe the cell electrophysiological dynamics,

$$
\begin{gathered}
\partial_{\mathrm{t}} \mathrm{V}_{\mathrm{m}}=-\mathrm{K}(-\Delta) \mathrm{V}_{\mathrm{m}}-\frac{1}{\mathrm{C}_{\mathrm{m}}} \mathrm{I}_{\text {ion }}, \\
\partial_{\mathrm{t}} \mathbf{y}=\mathbf{f}\left(\mathrm{V}_{\mathrm{m}}, \mathbf{y}\right), \quad \mathrm{I}_{\text {ion }}=\mathbf{g}\left(\mathrm{V}_{\mathrm{m}}, \mathbf{y}\right) .
\end{gathered}
$$

Here $I_{\text {ion }}$ is the ionic current through a number of different types of ion channels, $\mathbf{y}$ is a vector of ionic fluxes and their associated channel gating variable described by a suitable $\mathrm{ODE}, \mathrm{g}$ is the nonlinear function that relates the ionic fluxes to the total ionic current, $C_{m}$ is the capacitance of the cell membrane and $\mathrm{K}$ is the diffusion tensor. Modern cardiac electrophysiological models adapt the work of Hodgkin and Huxley [8] to describe the ion flux dynamics through a variety of ion channels using the idea of transition states between the differing conformations of the channels. Recently developed ion channel models have upwards of twenty different variables [6], and with finite element meshes of the human heart being of the order of millions of elements, it becomes quickly apparent that electrophysiological modelling of the human heart can be extremely computationally intensive, especially if the models are run over many heart beats.

Rather than using one of these complex cell models we illustrate our ideas by using one of the simplest single cell models, namely the FitzHugh-Nagumo model $[9,16]$. A dimensionless FitzHugh-Nagumo monodomain model is [22]

$$
\begin{aligned}
\frac{\partial v}{\partial t} & =\nabla \cdot(K \nabla v)+\mathrm{I}_{\text {ion }}(v, w) \\
\frac{\partial w}{\partial t} & =\mathrm{a} v-\mathrm{b} w+\mathrm{c} \\
\mathrm{I}_{\text {ion }} & =\frac{\mathrm{C}_{2}}{v_{\text {amp }}}\left(v-v_{\text {rest }}\right) w+\frac{\mathrm{C}_{1}}{v_{\text {amp }}^{2}}\left(v-v_{\text {rest }}\right)\left(v-v_{\text {th }}\right)\left(v-v_{\text {peak }}\right),
\end{aligned}
$$


where $v$ is a normalized transmembrane potential, $v_{\text {rest }}$ and $v_{\text {peak }}$ are the resting and peak potentials, respectively, with $v_{\text {amp }}=v_{\text {peak }}-v_{\text {rest }}$ and $v_{\text {th }}=$ $v_{\text {peak }}+v_{\text {rest }}$, and $w$ is a dimensionless time dependent recovery variable. It is known that this model has travelling wave solutions with an appropriate choice of parameters and stimulus [18]. The constants $\mathrm{a}, \mathrm{b}, \mathrm{c}, \mathrm{C}_{1}$ and $\mathrm{C}_{2}$ are all non-negative and standard values are 0.13, 0.013, 1, 0.26 and 0.1, respectively.

Bidomain and monodomain models represent the tissue as a continuum with space averaged properties. However, the extracellular space in which the cardiac myocytes are embedded is highly heterogeneous, consisting of ground substance, blood vessels, connective tissue, collagen and empty space [7]. Recent computational attempts to model this heterogeneity in cardiac tissue simulations, considered, for example, tissue models with empty spaces [17]. These models better replicated some characteristics of electrical propagation, such as the effect of excitable cells on wavefront velocity [15]. On the other hand, their numerical resolution is expensive as they involve extremely fine spatial discretizations at a subcellular level.

We propose a fundamental rethink of the homogenisation approach via the use of a fractional Fick's Law [11, 13, 14, 20] and, in particular, we introduce a fractional FitzHugh-Nagumo monodomain model in which we capture the spatial heterogeneities and spatial connectivities in the extra-cellular domain through the use of fractional derivatives. In two dimensions, characterized by the coordinates $(x, y)$ (i.e., a thin slice of tissue), possible formulations lead to a fractional model with the same fractional power in both spatial components, namely

$$
\frac{\partial v}{\partial t}=K^{\alpha} v+I_{\text {ion }}(v, w)
$$

with (4) and (5), or a model with different fractional powers $\alpha_{1}$ and $\alpha_{2}$ in the $x$ and $y$ directions, respectively, namely

$$
\frac{\partial v}{\partial t}=K_{x} \frac{\partial^{\alpha_{1}} v}{\partial|x|^{\alpha_{1}}}+K_{y} \frac{\partial^{\alpha_{2}} v}{\partial|y|^{\alpha_{2}}}+\mathrm{I}_{\text {ion }}(v, w)
$$


with (4) and (5). Here $1<\alpha_{1}, \alpha_{2} \leqslant 2, K_{x}$ and $K_{y}$ are diffusion coefficients and $\mathbf{R}^{\alpha}=\left(R_{x}^{\alpha}, R_{y}^{\alpha}\right)=\left(\partial^{\alpha} / \partial|x|^{\alpha}, \partial^{\alpha} / \partial|y|^{\alpha}\right)$ is a sequential Riesz fractional order operator in space [13]. The space Riesz fractional operators are defined on the whole space as, for example

$$
\frac{\partial^{\alpha_{1}} v}{\partial|x|^{\alpha_{1}}}=-\frac{1}{2 \cos \left(\pi \alpha_{1} / 2\right)}\left(\frac{\partial^{\alpha_{1}} v}{\partial x^{\alpha_{1}}}+\frac{\partial^{\alpha_{1}} v}{\partial(-\chi)^{\alpha_{1}}}\right),
$$

where

$$
\begin{aligned}
& \frac{\partial^{\alpha_{1}} v(x, y, t)}{\partial x^{\alpha_{1}}}=\frac{1}{\Gamma\left(2-\alpha_{1}\right)} \frac{\partial^{2}}{\partial x^{2}} \int_{-\infty}^{x} \frac{v(\xi, y, t)}{(x-\xi)^{\alpha_{1}-1}} d \xi \\
& \frac{\partial^{\alpha_{1}} v(x, y, t)}{\partial(-x)^{\alpha_{1}}}=\frac{1}{\Gamma\left(2-\alpha_{1}\right)} \frac{\partial^{2}}{\partial x^{2}} \int_{x}^{+\infty} \frac{v(\xi, y, t)}{(\xi-x)^{\alpha_{1}-1}} d \xi .
\end{aligned}
$$

Similar expressions are defined for the space Riesz fractional derivative of order $\alpha_{2}\left(1<\alpha_{2} \leqslant 2\right)$ with respect to $y$.

There are other modelling approaches that could be used to replace (3) with a fractional model. For example, we could define the operator $[\nabla \cdot(K \nabla)]^{\alpha / 2}$ by subordination [5]. However, this is not the same as using a fractional Fick's Law which leads to the formulation (7). The formulation (7) makes more sense in the context of cardiac electrophysiology. This is because in the heart, myocyte fibres tend to align in one particular direction and so the propagation of the electrical wave is different orthogonally, longitudinally and transversely along the fibres. Nevertheless, the wave has to travel through all components of heterogeneous tissue.

We choose zero Dirichlet boundary conditions as there are some subtleties with other types of boundary conditions for fractional models on finite domains [10]. In developing a numerical method for the coupled differential equations, we previously used a decoupled numerical technique [23, 24, 25]. Our fractional monodomain equation is solved by operator splitting, in which we first solve the fractional partial differential equation for $v$ and then the ODE for $w$, at each time step. In doing this our source term must be viewed as being locally 
Lipschitz and so, in Section 3, we deal with stability and convergence issues in this context.

An implicit numerical method with a shifted Grünwald-Letnikov approximation for the space-fractional problem is proposed in Section 2. The stability and convergence of this approach is discussed in Section 3, under the assumption that the source term is only locally Lipschitz. Some numerical results are given in Section 4 and discussions and conclusions are presented in Section 5.

\section{An implicit numerical method}

To develop our numerical method for the coupled fractional monodomain model, a decoupled numerical technique is used. In order to make our ideas clear, we assume $\alpha_{1}=\alpha_{2}=\alpha$, but our approach is equally valid in the case $\alpha_{1} \neq \alpha_{2}$. For given $v_{n}$ and $w_{n}$ at $t=t_{n}$, we solve

$$
\begin{aligned}
\frac{\partial v}{\partial t} & =K_{x} \frac{\partial^{\alpha} v}{\partial|x|^{\alpha}}+K_{y} \frac{\partial^{\alpha} v}{\partial|y|^{\alpha}}+I_{\text {ion }}\left(v_{n}, w_{n}\right), \\
I_{\text {ion }} & =\frac{C_{2}}{v_{\text {amp }}}\left(v_{n}-v_{\text {rest }}\right) w_{n}+\frac{C_{1}}{v_{\text {amp }}^{2}}\left(v_{n}-v_{\text {rest }}\right)\left(v_{n}-v_{\text {th }}\right)\left(v_{n}-v_{\text {peah }}\right),
\end{aligned}
$$

for $v=v_{n+1}$ at $t=t_{n+1}$. Then, for $w=w_{n+1}$ at $t=t_{n+1}$, given $v_{n+1}$ and $w_{n}$, we solve

$$
\frac{\partial w}{\partial t}=a v_{n+1}-b w+c .
$$

We cast our analysis as a two dimensional fractional Riesz space nonlinear reaction-diffusion model

$$
\frac{\partial u}{\partial t}=K_{x} \frac{\partial^{\alpha} u}{\partial|x|^{\alpha}}+K_{y} \frac{\partial^{\alpha} u}{\partial|y|^{\alpha}}+f(u, x, y, t),
$$

where $1<\alpha \leqslant 2$ and $0<K_{x}, 0<K_{y}$ are diffusion coefficients, and $f(u, x, y, t)$ is locally Lipschitz continuous. We use initial conditions

$$
u(x, y, 0)=\psi(x, y), \quad 0 \leqslant x \leqslant D_{x}, \quad 0 \leqslant y \leqslant D_{y},
$$


and zero Dirichlet boundary conditions

$$
u(0, y, t)=u\left(D_{x}, y, t\right)=u(x, 0, t)=u\left(x, D_{y}, t\right)=0,
$$

where the $(x, y)$ domain is $\left[0, D_{x}, 0, D_{y}\right]$. Baeumer et al. $[1,2]$ showed how to solve nonlinear reaction-diffusion equations of type (8) by an operator splitting method when the abstract function $f$ is only locally Lipschitz $[1,2,21]$.

Remark 1 . We say that $f: X \rightarrow X$ is globally Lipschitz continuous if for some $L>0$, we have $\|f(u)-f(v)\| \leqslant L\|u-v\|$ for all $u, v \in X$, and is locally Lipschitz continuous, if the latter holds for $\|u\|,\|v\| \leqslant M$ with $L=L(M)$ for any $M>0[1,2]$.

We assume that for all $k=1,2, \ldots, N,\left\|u\left(x, y, t_{k}\right)\right\|,\left\|v\left(x, y, t_{k}\right)\right\| \leqslant M_{k}$ for constant $M_{k}>0$, and

$$
\| f\left(u\left(x, y, t_{k}\right)-f\left(v\left(x, y, t_{k}\right)\right)\left\|\leqslant L_{k}\right\| u\left(x, y, t_{k}\right)-v\left(x, y, t_{k}\right) \|,\right.
$$

for all $(x, y) \in\left[0, D_{x}, 0, D_{y}\right]$, where we define $L_{k}=L\left(M_{k}\right)$ and $L_{\max }=$ $\max _{0 \leqslant k \leqslant N} L_{k}$.

For the numerical simulation of equation (8) over the time interval $[0, T]$, for integer $m_{1}$ and $m_{2}$, let $h_{x}=D_{x} / m_{1}, h_{y}=D_{y} / m_{2}$ and $\tau=T / N$ be the space and time grid sizes, respectively; $x_{i}=i h_{x}$ for $i=0,1, \ldots, m_{1} ; y_{j}=j h_{y}$ for $j=0,1, \ldots, m_{2}$. Define $\mathfrak{u}_{i, j}^{n}$ as the numerical approximation of $\mathfrak{u}(x, y, t)$ at $x=x_{i}, y=y_{j}, t=t_{n}$. The initial conditions are $u_{i, j}^{0}=\psi\left(x_{i}, y_{j}\right)$.

We use the shifted Grünwald-Letnikov scheme on the finite domain [12, 26] to discretise the Riesz fractional derivatives as

$$
\begin{gathered}
\left.\frac{\partial^{\alpha} u}{\partial x^{\alpha}}\right|_{\left(x_{i}, y_{j}, t_{n}\right)}=\frac{1}{\left(h_{x}\right)^{\alpha}} \sum_{l=0}^{i+1} g_{\alpha}^{(l)} u\left(x_{i-l+1}, y_{j}, t_{n}\right)+O\left(h_{x}\right), \\
\left.\frac{\partial^{\alpha} u}{\partial(-x)^{\alpha}}\right|_{\left(x_{i}, y_{j}, t_{n}\right)}=\frac{1}{\left(h_{x}\right)^{\alpha}} \sum_{l=0}^{m_{1}-i+1} g_{\alpha}^{(l)} u\left(x_{i+l-1}, y_{j}, t_{n}\right)+O\left(h_{x}\right) .
\end{gathered}
$$


A similar result holds in the $y$ direction. Here $g_{\alpha_{1}}^{(0)}=1$ and

$$
g_{\alpha}^{(l)}=(-1)^{l}\left(\begin{array}{c}
\alpha \\
l
\end{array}\right)=-g_{\alpha}^{(l-1)} \frac{\alpha-l+1}{l} .
$$

Lemma 2. [26, Lemma 3.3, p. 1768] The coefficients $\mathrm{g}_{\alpha}^{(\mathrm{l})}, \mathrm{l}=1,2, \ldots$, satisfy

- $\mathrm{g}_{\alpha}^{(0)}=1, \mathrm{~g}_{\alpha}^{(1)}=-\alpha<0$, and $\mathrm{g}_{\alpha}^{(\mathrm{l})}>0$ when $\mathrm{l} \neq 1$;

- $\sum_{l=0}^{\infty} \mathrm{g}_{\alpha}^{(\mathrm{l})}=0$, and $\sum_{\mathrm{l}=0}^{\mathrm{n}} \mathrm{g}_{\alpha}^{(\mathrm{l})}<0$ for $\mathrm{n}=1,2, \ldots$.

Lemma 3 (discrete Gronwall inequality). [26, Lemma 3.4, p. 1768] Suppose that $\mathrm{f}_{\mathrm{k}} \geqslant 0$ and $\eta_{\mathrm{k}} \geqslant 0$ for $\mathrm{k}=0,1,2, \ldots$, and

$$
\begin{aligned}
\eta_{k+1} & \leqslant\left(1+C_{0} \tau\right) \eta_{k}+\tau f_{k} \quad \text { for } k=0,1,2, \ldots, \\
\eta_{0} & =0
\end{aligned}
$$

where $\mathrm{C}_{0} \geqslant 0$ is constant, then $\eta_{k+1} \leqslant e^{\mathrm{C}_{0} t_{k}} \sum_{j=0}^{k} \tau f_{j}$.

Using the shifted Grünwald-Letnikov scheme, we obtain the discrete form of (8)

$$
\begin{aligned}
u\left(x_{i}, y_{j}, t_{n}\right)= & u\left(x_{i}, y_{j}, t_{n-1}\right) \\
& +r_{i, j}^{(1)}\left[\sum_{l=0}^{i+1} g_{\alpha}^{(l)} u\left(x_{i-l+1}, y_{j}, t_{n}\right)+\sum_{l=0}^{m_{1}-i+1} g_{\alpha}^{(l)} u\left(x_{i+l-1}, y_{j}, t_{n}\right)\right] \\
& +r_{i, j}^{(2)}\left[\sum_{l=0}^{j+1} g_{\alpha}^{(l)} u\left(x_{i}, y_{j-l+1}, t_{n}\right)+\sum_{l=0}^{m_{2}-j+1} g_{\alpha}^{(l)} u\left(x_{i}, y_{j+l-1}, t_{n}\right)\right] \\
& +\tau f\left[u\left(x_{i}, y_{j}, t_{n-1}\right), x_{i}, y_{j}, t_{n-1}\right]+R_{i, j, n},
\end{aligned}
$$

where $f\left[u\left(x_{i}, y_{j}, t_{n-1}\right), x_{i}, y_{j}, t_{n-1}\right] \equiv f_{i, j}^{n-1}, c_{\alpha}=1 /[2 \cos (\pi \alpha / 2)], r_{i, j}^{(1)}=$ $\left[\tau K_{x}\left(x_{i}, y_{j}\right) c_{\alpha}\right] / h_{x}^{\alpha}, r_{i, j}^{(2)}=\left[\tau K_{y}\left(x_{i}, y_{j}\right) c_{\alpha}\right] / h_{y}^{\alpha}$, and $\left|R_{i, j, n}\right| \leqslant C^{*}\left(\tau^{2}+\tau h_{x}+\tau h_{y}\right)$ for constant $\mathrm{C}^{*}$. 
The implicit numerical method is

$$
\begin{aligned}
u_{i, j}^{n} & -r_{i, j}^{(1)}\left[\sum_{l=0}^{i+1} g_{\alpha}^{(l)} u_{i-l+1, j}^{n}+\sum_{l=0}^{m_{1}-i+1} g_{\alpha}^{(l)} u_{i+l-1, j}^{n}\right] \\
- & r_{i, j}^{(2)}\left[\sum_{l=0}^{j+1} g_{\alpha}^{(l)} u_{i, j-l+1}^{n}+\sum_{l=0}^{m_{2}-j+1} g_{\alpha}^{(l)} u_{i, j+l-1}^{n}\right]=u_{i, j}^{n-1}+\tau f_{i, j}^{n-1},
\end{aligned}
$$

with

$$
\begin{aligned}
& u_{i, j}^{0}=\psi_{i, j}=\psi\left(x_{i}, y_{j}\right), \\
& u_{0, j}^{n}=u_{m_{1}, j}^{n}=u_{i, 0}^{n}=u_{i, m_{2}}^{n}=0 .
\end{aligned}
$$

Or, in matrix form

$$
A^{(n)} \mathbf{u}^{(n)}=\mathbf{b}^{(n)},
$$

where $\mathbf{u}^{(\mathfrak{n})}=\left(\mathfrak{u}_{1,1}^{\mathfrak{n}}, \mathfrak{u}_{1,2}^{n}, \ldots, \mathfrak{u}_{1, m_{2}-1}^{\mathfrak{n}}, \mathfrak{u}_{2,1}^{n}, \ldots, \mathfrak{u}_{\mathfrak{m}_{1}-1, m_{2}-1}^{\mathfrak{n}}\right), \mathbf{b}^{(\mathfrak{n})}$ is a $\left(m_{1}-\right.$ 1) $\left(m_{2}-1\right)$ dimensional column vector that includes the known initial and boundary values, the known source term values and the previous $n$ layer known values, and the coefficient matrix $A^{(\mathfrak{n})}$ is a $\left(m_{1}-1\right)\left(m_{2}-1\right) \times\left(m_{1}-\right.$ 1) $\left(m_{2}-1\right)$ known matrix.

Gauss-Seidel iteration technique [19] is used to solve the implicit difference scheme (15)-(17) or (18).

\section{$3 \quad$ Stability and convergence}

We first prove the stability of (15)-(17). Let $\widetilde{\mathfrak{u}}_{i, j}^{n}$ be the approximate solution of (15)-(17) and $\varepsilon_{i, j}^{n}=u_{i, j}^{n}-\widetilde{u}_{i, j}^{n}$ denote the corresponding error with $E^{n}=$ $\left(\varepsilon_{1,1}^{\mathfrak{n}}, \varepsilon_{2,1}^{\mathfrak{n}}, \ldots, \varepsilon_{\mathrm{m}_{1}-1, \mathrm{~m}_{2}-1}^{\mathfrak{n}}\right)$. Assuming

$$
\left|\varepsilon_{i_{0}, j_{0}}^{n}\right|=\max _{1 \leqslant i \leqslant m_{1}-1,1 \leqslant j \leqslant m_{2}-1}\left|\varepsilon_{i, j}^{n}\right|=\left\|E^{n}\right\|_{\infty}
$$

we have the following theorem. 
Theorem 4. The implicit numerical method (15)-(17) is unconditionally stable, and

$$
\left\|\mathrm{E}^{\mathrm{n}}\right\|_{\infty} \leqslant \mathrm{C}\left\|\mathrm{E}^{0}\right\|_{\infty} \quad \text { for } \mathrm{n}=0,1,2, \ldots, \mathrm{N}
$$

where $\mathrm{C}$ is a positive number independent of $\mathrm{h}_{\mathrm{x}}, \mathrm{h}_{\mathrm{y}}$ and $\tau$.

Proof: According to (15)-(17), the error $\varepsilon_{i, j}^{n}$ satisfies

$$
\begin{aligned}
& \varepsilon_{i, j}^{n}-r_{i, j}^{(1)}\left[\sum_{l=0}^{i+1} g_{\alpha}^{(l)} \varepsilon_{i-l+1, j}^{n}+\sum_{l=0}^{m_{1}-i+1} g_{\alpha}^{(l)} \varepsilon_{i+l-1, j}^{n}\right] \\
& -r_{i, j}^{(2)}\left[\sum_{l=0}^{j+1} g_{\alpha}^{(l)} \varepsilon_{i, j-l+1}^{n}+\sum_{l=0}^{m_{2}-j+1} g_{\alpha}^{(l)} \varepsilon_{i, j+l-1}^{n}\right] \\
& =\varepsilon_{i, j}^{n-1}+\tau f\left(u_{i, j}^{n-1}, x_{i}, y_{j}, t_{n-1}\right)-\tau f\left(\widetilde{u}_{i, j}^{n-1}, x_{i}, y_{j}, t_{n-1}\right) .
\end{aligned}
$$

From Lemma 2 and (20),

$$
\begin{aligned}
\left\|E^{n}\right\|_{\infty} \leqslant & \mid \varepsilon_{i_{0}, j_{0}}^{n}-r_{i_{0}, j_{0}}^{(1)}\left[\sum_{l=0}^{i+1} g_{\alpha}^{(l)} \varepsilon_{i_{0}-l+1, j_{0}}^{n}+\sum_{l=0}^{m_{1}-i_{0}+1} g_{\alpha}^{(l)} \varepsilon_{i_{0}+l-1, j}^{n}\right] \\
& -r_{i_{0}, j_{0}}^{(2)}\left[\sum_{l=0}^{j_{0}+1} g_{\alpha}^{(l)} \varepsilon_{i_{0}, j_{0}-l+1}^{n}+\sum_{l=0}^{m_{2}-j_{0}+1} g_{\alpha}^{(l)} \varepsilon_{i_{0}, 0+l-1}^{n}\right] \mid \\
= & \left|\varepsilon_{i_{0}, j_{0}}^{n-1}+\tau f\left(u_{i, j}^{n-1}, x_{i}, y_{j}, t_{n-1}\right)-\tau f\left(\widetilde{u}_{i, j}^{n-1}, x_{i}, y_{j}, t_{n-1}\right)\right| \\
\leqslant & \left(1+\tau L_{n-1}\right)\left\|E^{n-1}\right\|_{\infty} \\
\leqslant & \prod_{k=0}^{n-1}\left(1+\tau L_{k}\right)\left\|E^{0}\right\|_{\infty} \\
\leqslant & \left(1+\tau L_{\max }\right)^{n}\left\|E^{0}\right\|_{\infty} \\
\leqslant & e^{n \tau \tau L_{\max }}\left\|E^{0}\right\|_{\infty} \\
\leqslant & e^{L_{\max } \top}\left\|E^{0}\right\|_{\infty}=C E^{0} \|_{\infty} .
\end{aligned}
$$


Now we consider the convergence of the implicit numerical method.

Theorem 5. Assume that the continuous problem (8)-(10) has a smooth solution $\mathfrak{u}\left(\mathrm{x}_{\mathfrak{i}}, \mathrm{y}_{\mathrm{j}}, \mathrm{t}_{\mathrm{n}}\right)$ and $\mathrm{u}_{\mathrm{i}, \mathrm{j}}^{\mathrm{n}}$ is the solution of the implicit numerical method (15)(17) at mesh point $\left(x_{i}, y_{j}, t_{n}\right)$. Then there is a positive constant $C$ independent of $h_{x}, h_{y}$ and $\tau$, such that for all $i, j$ and $n$

$$
\left|u\left(x_{i}, y_{j}, t_{n}\right)-u_{i, j}^{n}\right| \leqslant C\left(\tau+h_{x}+h_{y}\right) .
$$

Proof: Define $\eta_{i, j}^{n}=u\left(x_{i}, y_{j}, t_{n}\right)-u_{i, j}^{n}$ and $Y^{n}=\left(\eta_{1,1}^{n}, \eta_{2,1}^{n}, \ldots, \eta_{m_{1}-1, m_{2}-1}^{n}\right)$. Then

$$
\begin{aligned}
& \eta_{i, j}^{n}-r_{i, j}^{(1)}\left[\sum_{l=0}^{i+1} g_{\alpha}^{(l)} \eta_{i-l+1, j}^{n}+\sum_{l=0}^{m_{1}-i+1} g_{\alpha}^{(l)} \eta_{i+l-1, j}^{n}\right] \\
& -r_{i, j}^{(2)}\left[\sum_{l=0}^{j+1} g_{\alpha}^{(l)} \eta_{i, j-l+1}^{n}+\sum_{l=0}^{m_{2}-j+1} g_{\alpha}^{(l)} \eta_{i, j+l-1}^{n}\right] \\
& =\eta_{i, j}^{n-1}+\tau f\left[u\left(x_{i}, y_{j}, t_{n-1}\right), x_{i}, y_{j}, t_{n-1}\right]-\tau f\left(u_{i, j}^{n}, x_{i}, y_{j}, t_{n-1}\right)+R_{i, j, n} .
\end{aligned}
$$


Using Lemma 2, (21) and the discrete Gronwall inequality from Lemma 3,

$$
\begin{aligned}
\left\|Y^{n}\right\|_{\infty} \leqslant & \mid \eta_{i_{0}, j_{0}}^{n}-r_{i_{0}, j_{0}}^{(1)}\left[\sum_{l=0}^{i+1} g_{\alpha}^{(l)} \eta_{i_{0}-l+1, j_{0}}^{n}+\sum_{l=0}^{m_{1}-i_{0}+1} g_{\alpha}^{(l)} \eta_{i_{0}+l-1, j}^{n}\right] \\
& -r_{i_{0}, j_{0}}^{(2)}\left[\sum_{l=0}^{j_{0}+1} g_{\alpha}^{(l)} \eta_{i_{0}, j_{0}-l+1}^{n}+\sum_{l=0}^{m_{2}-j_{0}+1} g_{\alpha}^{(l)} \eta_{i_{0}, j_{0}+l-1}^{n}\right] \mid \\
= & \mid \eta_{i_{0}, j_{0}}^{n-1}+\tau f\left[u\left(x_{i_{0}}, y_{j_{0}}, t_{n-1}\right), x_{i_{0}}, y_{j_{0}}, t_{n-1}\right]-\tau f\left(u_{i_{0}, j_{0}}^{n-1}, x_{i_{0}}, y_{j_{0}}, t_{n-1}\right) \\
& +R_{i_{0}, j_{0}, n} \mid \\
\leqslant & \left(1+\tau L_{n-1}\right)\left\|Y^{n-1}\right\|_{\infty}+C^{*}\left(\tau^{2}+\tau h_{x}+\tau h_{y}\right) \\
\leqslant & \left(1+\tau L_{\max }\right)\left\|Y^{n-1}\right\|_{\infty}+C^{*}\left(\tau^{2}+\tau h_{x}+\tau h_{y}\right) \\
\leqslant & \left(1+\tau L_{\max }\right)^{n}\left\|Y^{0}\right\|_{\infty}+\sum_{k=0}^{n-1}\left(1+\tau L_{m a x}\right)^{k} C^{*}\left(\tau^{2}+\tau h_{x}+\tau h_{y}\right) \\
\leqslant & \left(1+\tau L_{\max }\right)^{n} n \tau C^{*}\left(\tau+h_{x}+h_{y}\right) \\
\leqslant & e^{n \tau L_{\max }} T C^{*}\left(\tau+h_{x}+h_{y}\right) \\
= & C\left(\tau+h_{x}+h_{y}\right) .
\end{aligned}
$$

We see that for any $x, y$ and $t$, if $h_{x}, h_{y}$ and $\tau$ approach zero such that $\left(i h_{x}, j h_{y}, n \tau\right) \rightarrow(x, y, t)$, then $u_{i, j}^{n}$ approaches $u(x, y, t)$. This proves that $u_{i, j}^{n}$ converges to $u\left(x_{i}, y_{j}, t_{n}\right)$ as $h_{x}, h_{y}$ and $\tau$ tend to zero.

\section{Numerical results}

In this section, some numerical results are presented to confirm our theoretical analysis from Section 3. Firstly, the implicit numerical method introduced in Section 2, is employed to simulate a two dimensional fractional FitzHughNagumo monodomain model [4]. 
Example 6. The FitzHugh-Nagumo monodomain model [4] is

$$
\begin{aligned}
& \frac{\partial u}{\partial t}=K_{x} \frac{\partial^{\alpha_{1}} u}{\partial|x|^{\alpha_{1}}}+K_{y} \frac{\partial^{\alpha_{2}} u}{\partial|y|^{\alpha_{2}}}+u(1-u)(u-a)-v, \\
& \frac{\partial v}{\partial t}=\varepsilon(\beta u-\gamma v-\delta),
\end{aligned}
$$

where $a=0.1, \varepsilon=0.01, \beta=0.5, \gamma=1, \delta=0$, which is known to generate stable patterns in the system in the form of re-entrant spiral waves. The trivial state $(u, v)=(0,0)$ was perturbed by setting the lower left quarter of the domain to $u=1$ and the upper half to $v=0.1$, which allows the initial condition to curve and rotate clockwise generating the spiral pattern. For $\mathrm{D}_{x}=2.5$ and $\mathrm{D}_{y}=2.5$, this leads to the initial conditions

$$
\begin{aligned}
& u(x, y, 0)= \begin{cases}1.0, & 0<x \leqslant 1.25,0<y<1.25 \\
0.0, & 1.25 \leqslant x<2.5,0<y<1.25 \\
0.0, & 0<x \leqslant 1.25,1.25 \leqslant y<2.5 \\
0.0, & 1.25 \leqslant x<2.5,1.25 \leqslant y<2.5\end{cases} \\
& v(x, y, 0)= \begin{cases}0.0, & 0<x \leqslant 1.25,0<y<1.25 \\
0.0, & 1.25 \leqslant x<2.5,0<y<1.25 \\
0.1, & 0<x \leqslant 1.25,1.25 \leqslant y<2.5 \\
0.1, & 1.25 \leqslant x<2.5,1.25 \leqslant y<2.5\end{cases}
\end{aligned}
$$

with zero Dirichlet boundary conditions

$$
u(0, y, t)=u(2.5, y, t)=0, \quad u(x, 0, t)=u(x, 2.5, t)=0 .
$$

The domain is discretised using $\mathrm{m}_{1,2}=256$ points in each spatial coordinate, with a time step $\tau=0.1$.

Spiral waves of the stable rotating solution in the Fitzhugh-Nagumo mode, $\alpha_{1}=\alpha_{2}=2$, with $K_{x}=K_{y}=10^{-4}$ and $K_{x}=K_{y}=10^{-5}$ are shown in Figure 1, whereas the behaviour of the Riesz space fractional FitzhughNagumo model with $\alpha_{1}=\alpha_{2}=1.7, \alpha_{1}=\alpha_{2}=1.5$ and $K_{x}=K_{y}=10^{-4}$ is shown in Figure 2. We see that the width of the excitation wavefront is 
(a)


Figure 1: Spiral waves in the Fitzhugh-Nagumo model at $t=1000$ with $\alpha_{1}=\alpha_{2}=2:$ (a) $K_{x}=K_{y}=10^{-4}$; (b) $K_{x}=K_{y}=10^{-5}$.

markedly reduced for decreasing fractional power, as is the wavelength of the system, with the domain able to accommodate a larger number of wavefronts with smaller fractional powers.

We emphasise that reducing the fractional power is not equivalent to a decreased diffusion coefficient in the pure diffusion case. This is clearly observed by comparing Figure 1(b) and Figure 2(b).

For anisotropic diffusion ratios $\mathrm{K}_{\mathrm{x}}=10^{-4}, \mathrm{~K}_{\mathrm{y}} / \mathrm{K}_{\mathrm{x}}=0.25<1$ and $\mathrm{K}_{\mathrm{y}}=10^{-4}$, $\mathrm{K}_{\mathrm{x}} / \mathrm{K}_{\mathrm{y}}=0.25<1$, wave propagation at $\mathrm{t}=1000$ in the space Riesz fractional Fitzhugh-Nagumo model with zero Dirichlet boundary conditions is shown in Figure 3. The spiral wave now follows an elliptical pattern. Anisotropic fractional ratios $\alpha_{1}=2, \alpha_{2} / \alpha_{1}=0.825<1$ and $\alpha_{2}=2, \alpha_{1} / \alpha_{2}=0.825<1$ exert a contrasting effect on the curvature of the solutions, see Figure 4, reflecting a distinct super-diffusion scale in each of the spatial dimensions of the system.

Example 7. To demonstrate the effectiveness of our numerical method's terms of convergence, we now present an example that exhibits an exact solution. Consider the two dimensional fractional Riesz space nonlinear 
(a)

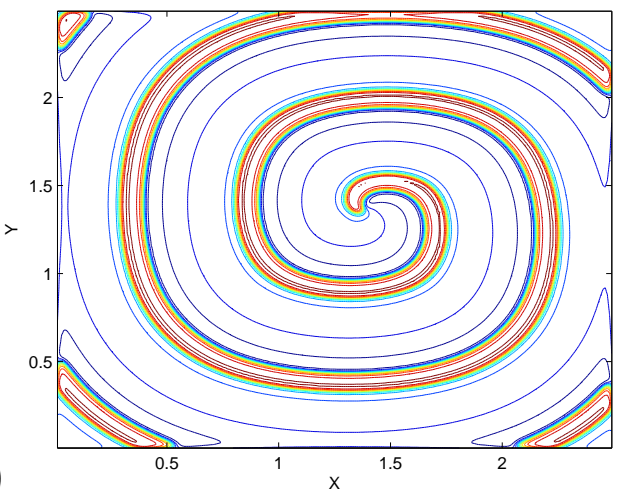

(b)



Figure 2: Spiral waves in the Fitzhugh-Nagumo model at $t=1000$ with $\mathrm{K}_{\mathrm{x}}=\mathrm{K}_{\mathrm{y}}=10^{-4}$ : (a) $\alpha_{1}=\alpha_{2}=1.7$; (b) $\alpha_{1}=\alpha_{2}=1.5$.

(a)

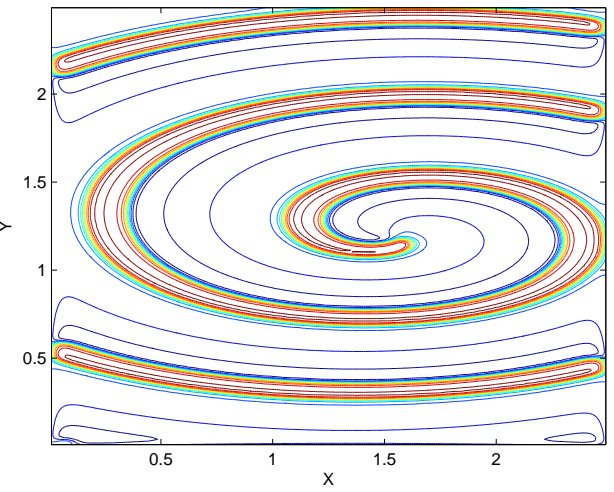

(b)

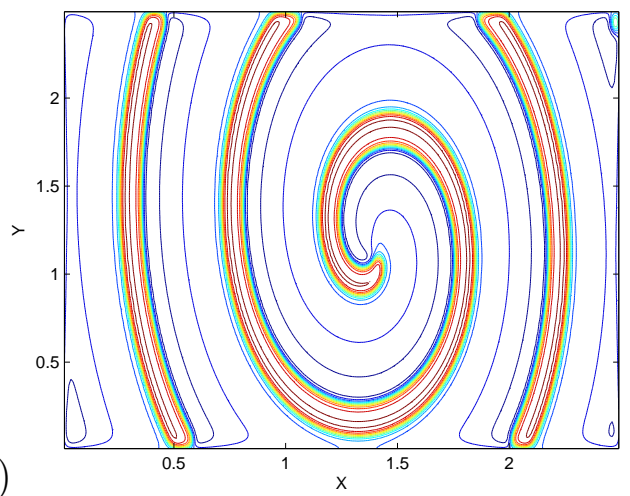

Figure 3: Spiral waves in the Fitzhugh-Nagumo model at $t=1000$ with $\alpha_{1}=\alpha_{2}=2$ : (a) $\mathrm{K}_{\mathrm{x}}=10^{-4}, \mathrm{~K}_{\mathrm{x}} / \mathrm{K}_{\mathrm{y}}=0.25$; (b) $\mathrm{K}_{\mathrm{y}}=10^{-4}, \mathrm{~K}_{\mathrm{x}} / \mathrm{K}_{\mathrm{y}}=0.25$. 
(a)

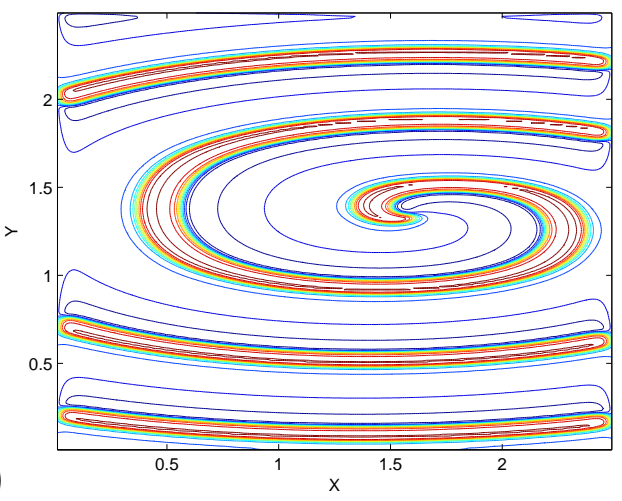

(b)



Figure 4: Spiral waves in the Fitzhugh-Nagumo model at $t=1000$ with $\mathrm{K}_{\mathrm{x}}=\mathrm{K}_{\mathrm{y}}=10^{-4}$ : (a) $\alpha_{1}=2, \alpha_{2} / \alpha_{1}=0.825$; (b) $\alpha_{2}=2, \alpha_{1} / \alpha_{2}=0.825$.

reaction-diffusion model

$$
\frac{\partial u}{\partial t}=K\left(\frac{\partial^{\alpha} u}{\partial|x|^{\alpha}}+\frac{\partial^{\alpha} u}{\partial|y|^{\alpha}}\right)+f(u, x, y, t),
$$

with initial condition $\mathfrak{u}(x, y, 0)=0$ and zero Dirichlet boundary conditions, where

$$
\begin{aligned}
f(u, x, y, t)= & -\frac{K t^{1+\alpha}}{2 \cos (\alpha \pi / 2)}\left[y ^ { 2 } ( 1 - y ) ^ { 2 } \left(\frac{2\left[x^{2-\alpha}+(1-x)^{2-\alpha}\right]}{\Gamma(3-\alpha)}\right.\right. \\
& \left.-\frac{12\left[x^{3-\alpha}+(1-x)^{3-\alpha}\right]}{\Gamma(4-\alpha)}+\frac{24\left[x^{4-\alpha}+(1-x)^{4-\alpha}\right]}{\Gamma(5-\alpha)}\right) \\
& +x^{2}(1-x)^{2}\left(\frac{2\left[y^{2-\alpha}+(1-y)^{2-\alpha}\right]}{\Gamma(3-\alpha)}-\frac{12\left[y^{3-\alpha}+(1-y)^{3-\alpha}\right]}{\Gamma(4-\alpha)}\right. \\
& \left.\left.+\frac{24\left[y^{4-\alpha}+(1-y)^{4-\alpha}\right]}{\Gamma(5-\alpha)}\right)\right]+(1+\alpha) t^{\alpha} x^{2}(1-x)^{2} y^{2}(1-y)^{2}
\end{aligned}
$$

and $1<\alpha \leqslant 2,(x, y) \in[0,1] \times[0,1]$. 
Table 1: Maximum error behavior for the implicit numerical method (15)-(17) with $\tau=0.01$ as the grid size is reduced in Example 7 at time $T=1.0$.

\begin{tabular}{cc}
$h_{x}=h_{y}=h$ & $\left\|Y^{n}\right\|_{\infty}$ \\
\hline $1 / 8$ & $7.7 \times 10^{-5}$ \\
$1 / 16$ & $1.1 \times 10^{-5}$ \\
$1 / 32$ & $8.6 \times 10^{-6}$
\end{tabular}

The exact solution of the above problem is $u(x, y, t)=t^{1+\alpha} x^{2}(1-x)^{2} y^{2}(1-y)^{2}$, which is verified by substituting directly into (8). From Table 1 it is seen that our implicit numerical method is in good agreement with the exact solution and has a convergence rate in agreement with Theorem 4 .

\section{Discussion and Conclusions}

In cardiac electrophysiology, the action potential duration (APD), defined as the difference between repolarisation and activation times, is an important biomarker of proarrhyhmic risk. An important mechanism of an intact heart is the shortening of APD during propagation of the electrical wave in tissue. An important experimental study was performed by Badie and Bursac [3] in neonatal rat cell cultures, in which an isotropic monolayer of cells were grown in a circular dish and, after stimulation at the centre, large changes were recorded in the distribution of APD from the centre to the edge. Standard monodomain models cannot replicate this change in APD as a function of the distance from the stimulation at the centre of the dish. Consequently, BuenoOrovioy et al. [5] performed a comprehensive study comparing experimental data and a fractional monodomain model based on the fractional Laplacian.

We proposed a new monodomain formulation aimed at capturing the heterogeneity and complex connectivity patterns of tissue via a fractional FitzHughNagumo monodomain model. The model consists of a coupled fractional Riesz space nonlinear reaction-diffusion model and an ordinary differential 
equation. We used a decoupling technique and an implicit numerical method to solve the two dimensional problem, with Dirichlet boundary conditions, and the stability and convergence properties were analysed in detail. Although the source term is not globally Lipschitz continuous, the solution of the discrete numerical method still yields bounds on the solution of the continuous problem and the solution of the numerical method converges to the unique solution of the continuous problem as the time and space steps tend to zero $[1,2,21]$. The numerical results demonstrate the effectiveness of this approach and suggest that such models can have very different dynamics to the standard monodomain models and, as such, represent a powerful modeling approach for understanding the many aspects of electrophysiological dynamics in heterogeneous cardiac tissue, as well as other areas. We hope to study the consequences of this new approach to cardiac electrophysiology in future work.

Acknowledgements This research is supported by the Australian Research Council grant DP120103770. We thank the referees for their constructive comments and suggestions.

\section{References}

[1] B. Baeumer, M. Kovály, and M. M. Meerschaert, Fractional reproduction-dispersal equations and heavy tail dispersal kernels, Bulletin of Mathematical Biology 69:2281-2297, 2007. doi:10.1007/s11538-007-9220-2 C614, C625

[2] B. Baeumer, M. Kovály, and M. M. Meerschaert, Numerical solutions for fractional reaction-diffusion equations, Computers and Mathematics with Applications 55:2212-2226, 2008. doi:10.1016/j.camwa.2007.11.012 C614, C625 
[3] N. Badie and N. Bursac, Novel micropatterned cardiac cell cultures with realistic ventricular microstructure, Biophys J 96:3873-3885, 2009. doi:10.1016/j.bpj.2009.02.019 C624

[4] A. Bueno-Orovio, D. Kay, K. Burrage, Fourier spectral methods for fractional-in-space reaction-diffusion equations, Technical report, University of Oxford, 2013. C619, C620

[5] A. Bueno-Orovioy, D. Kay, V. Grau, B. Rodriguez and K. Burrage, Fractional dffusion models of electrical propagation in cardiac tissue: electrotonic effects and the modulated dispersion of repolarization, Technical report, University of Oxford, 2013. C612, C624

[6] K. F. Decker, J. Heijman, J. R. Silva, T. J. Hund and Y. Rudy, Properties and ionic mechanisms of action potential adaptation, restitution, and accommodation in canine epicardium, Am. J. Physiol Heart Circ. Physiol. 296:H1017-H1026, 2009.

doi:10.1152/ajpheart.01216.2008 C610

[7] J. S. Frank and G. A. Langer, The myocardial interstitium: its structure and its role in ionic exchange, J Cell Biol 60:586-601, 1974. doi:10.1083/jcb.60.3.586 C611

[8] A. L. Hodgkin and A. F. Huxley, A quantitative description of membrane current and its application to conduction and excitation in nerve, J. Physiol. (Lond), 117:500-544, 1952.

http://jp.physoc.org/content/117/4/500.html C610

[9] R. FitzHugh, Impulses and Physiological States in Theoretical Models of Nerve Membrane, Biophys. J., 1:445-466, 1961. doi:10.1016/S0006-3495(61)86902-6 C610

[10] D. Kay, I. W. Turner, N. Cusimano and K. Burrage, Reflections from a boundary: reflecting boundary conditions for space-fractional partial differential equations on bounded domains, Technical report, University of Oxford, 2013. . C612 
[11] F. Liu, V. Anh and I. Turner, Numerical solution of space fractional Fokker-Planck equation. J. Comp. and Appl. Math., 166:209-219, 2004. doi:10.1016/j.cam.2003.09.028 C611

[12] F. Liu, P. Zhuang, V. Anh and I. Turner and K. Burrage, Stability and convergence of the difference methods for the space-time fractional advection-diffusion equation. Appl. Math. Comp., 191:12-20, 2007. doi:10.1016/j.amc.2006.08.162 C614

[13] R. Magin, O. Abdullah, D. Baleanu and X. Zhou, Anomalous diffusion expressed through fractional order differential operators in the Bloch-Torrey equation, Journal of Magnetic Resonance 190:255-270, 2008. doi:10.1016/j.jmr.2007.11.007 C611, C612

[14] M. M. Meerschaert, J. Mortensenb and S. W. Wheatcraft, Fractional vector calculus for fractional advection-dispersion, Physica A, 367:181-190, 2006. doi:10.1016/j.physa.2005.11.015 C611

[15] L. C. McSpadden, R. D. Kirkton and N. Bursac, Electrotonic loading of anisotropic cardiac monolayers by unexcitable cells depends on connexin type and expression level, Am. J. Physiol. Cell Physiol. 297:C339-C351, 2009. doi:10.1152/ajpcell.00024.2009 C611

[16] J. Nagumo, S. Arimoto, and S. Yoshizawa, An active pulse transmission line simulating nerve axon, Proceedings of the IRE, 50:2061-2070, 1962. doi:10.1109/JRPROC.1962.288235 C610

[17] S. F. Roberts, J. G. Stinstra and C. S. Henriquez, Effect of nonuniform interstitial space properties on impulse propagation: a discrete multidomain model, Biophys J 95:3724-3737, 2008. doi:10.1529/biophysj.108.137349 C611

[18] J. Sundnes, G. T. Lines, X. Cai, B. F. Nielsen, K. A. Mardal and A. Tveitio, Computing the electrical activity in the heart, Springer-Verlag, 2006. C611 
[19] G. D. Smith, Numerical Solution of Partial Differential Equations: Finite Difference Methods, Clarendon Press, Oxford, 1985. C616

[20] F. J. Valdes-Parada, J. A. Ochoa-Tapia and J. Alvarez-Ramirez, Effective medium equations for fractional Fick law in porous media, Physica A, 373:339-353, 2007. doi:10.1016/j.physa.2006.06.007 C611

[21] Q. Yang, F. Liu and I. Turner, Stability and convergence of an effective numerical method for the time-space fractional Fokker-Planck equation with a nonlinear source term, International Journal of Differential Equations, 2010:464321, 2010, doi:10.1155/2010/464321 C614, C625

[22] W. Ying, A multilevel adaptive approach for computational cardiology, PhD thesis, Duke University, 2005. C610

[23] Q. Yu, F. Liu, I. Turner and K. Burrage, A computationally effective alternating direction method for the space and time fractional Bloch-Torrey equation in 3-D, Appl. Math. Comp., 219:4082-4095, 2012. doi:10.1016/j.amc.2012.10.056 C612

[24] Q. Yu, F. Liu, I. Turner and K. Burrage, Stability and convergence of an implicit numerical method for the space and time fractional Bloch-Torrey equation, the special issue of Fractional Calculus and Its Applications in-Philosophical Transactions of the Royal Society A: Mathematical, Physical and Engineering Sciences, 371:20120150, 2013. doi:10.1098/rsta.2012.0150 C612

[25] Q. Yu, F. Liu, I. Turner and K. Burrage, Numerical simulation of the fractional Bloch equations, J. Comp. Appl. Math., 255:635-651, 2014. doi:10.1016/j.cam.2013.06.027 C612

[26] P. Zhuang, F. Liu, V. Anh and I. Turner, Numerical methods for the variable-order fractional advection-diffusion equation with a nonlinear source term, SIAM J. Num. Anal., 47:1760-1781, 2009. doi:10.1137/080730597 C614, C615 


\section{Author addresses}

1. F. Liu, School of Mathematical Sciences, Queensland University of Technology, Brisbane, Qld. 4001, Australia. mailto:f.liu@qut.edu.au

2. I. Turner, School of Mathematical Sciences, Queensland University of Technology, Brisbane, Qld. 4001, Australia.

3. V. Anh, School of Mathematical Sciences, Queensland University of Technology, Brisbane, Qld. 4001, Australia.

4. Q. Yang, School of Mathematical Sciences, Queensland University of Technology, Brisbane, Qld. 4001, Australia.

5. K. Burrage, School of Mathematical Sciences, Queensland University of Technology, Brisbane, Qld. 4001, Australia;

Department of Computer Science and OCISB, University of Oxford, OXI 3QD, UK. 